\title{
Medication-Related Osteonecrosis of the Jaws (MRONJ) Induced by Incompatible Complete Removable Denture: A Case Report
}

\author{
Gelengül Gürbüz-Urvasızoğlu ${ }^{1}$, Nesrin $\operatorname{Saruhan}^{2}$, Mert Ataol $^{1, \text { * }}$ \\ ${ }^{1}$ Department of Oral and Maxillofacial Surgery Faculty of Dentistry, Ataturk University, Erzurum, Turkey \\ ${ }^{2}$ Department of Oral and Maxillofacial Surgery, Faculty of Dentistry, Eskişehir Osmangazi University, Eskişehir, Turkey
}

Email address:

gelengul2568@hotmail.com (G. Gürbüz-Urvasızoğlu),dt_nesrin@yahoo.com (N. Saruhan), ataolmert@gmail.com (M. Ataol)

${ }^{*}$ Corresponding author

\section{To cite this article:}

Gelengül Gürbüz-Urvasızoğlu, Nesrin Saruhan, Mert Ataol. Medication-Related Osteonecrosis of the Jaws (MRONJ) Induced by Incompatible Complete Removable Denture: A Case Report. International Journal of Clinical Oral and Maxillofacial Surgery. Vol. 3, No. 2, 2017, pp. 7-10. doi: 10.11648/j.ijcoms.20170302.11

Received: July 26, 2017; Accepted: September 12, 2017; Published: October 17, 2017

\begin{abstract}
Multiple myeloma is a malignant, hematological neoplasia affected plasma cells. It causes anemia, leads to increased susceptibility to infections by compromising immune response and severe pain is raised as a result of osteolytic lesions. For medical therapy of this condition, bisphosphonates (BPs) can be prescribed. Despite its great benefits in Multiple Myeloma (MM), in particular intravenous administration of BPs can lead to Medication-related osteonecrosis of the jaws (MRONJ). A 61-year-old female patient refers to our department for complaint of difficulty on usage of her prosthesis. In medical history, it was learned that her chemotherapy for multiple myeloma has been continued for 5 years and skull radiographs showed characteristic punched-out lytic lesions. With clinical and radiological examinations, this condition diagnosed as Stage II MRONJ. The aim of this study is to report the case of MRONJ within the reason of incompatible prosthesis by this way to be directive about preventive management.
\end{abstract}

Keywords: Bisphosphonate-Related Osteonecrosis of the Jaw, Denture, Dental Prosthesis, Multiple Myeloma, Medication-Related Osteonecrosis of the Jaw, Osteonecrosis, Zoledronic Acid

\section{Introduction}

Multiple myeloma (MM) describes as a malignant, hematological neoplasia of plasma cells. This disease affecting older adults, the mean age of patients is 65 years, and $\mathrm{MM}$ is more common in males than females. [1] This disease causes symptoms associated with anemia, a compromised immune response for this reason increased susceptibility to infections, osteolytic lesions and severe pain. [2] Cause of these multiple factors, bone destruction occurs in $90 \%$ of MM patients. [3] Bone destruction can result in 'skeletal related events' including bone pain, pathological fractures, spinal cord compression, and hypercalcemia. [4, 5]

For medical therapy of this condition, bisphosphonates (BPs) are prescribed for solid tumors and bone metastasis, hypercalcemia, Waldenstrom's macroglobulinemia, osteoporosis, Paget's disease and MM. [6] For MM patients,
BP therapy is used for persistent or severe hypercalcemia management and inhibit new bone destruction. BPs are recommended as ongoing therapy for all myeloma patients with bone disease. According to "Myeloma Management Guidelines - Consensus Report from the Scientific Advisors of the International Myeloma Foundation" [7]; the bisphosphonates are in general use for MM are pamidronate, zoledronic acid, and clodronate. These medications have proven to be equivalent in reducing skeletal related events or bone complications, such as fractures and pain.

Monitoring of BPs has a great importance. Renal toxicity is a concern with all i.v. bisphosphonates, especially with chronic users at least 2 years. Serum creatinine level must be measured before each administration of i.v. BPs. An increase of $>0.5$ $\mathrm{mg} / \mathrm{dl}$ may require dose/schedule adjustments. Periodic urine protein measurement (24 hr) is required (3-6 months) with chronic administration. One of the appropriate protections is to 
extend the infusion time (4 mg infusion - 45 minutes for zoledronic acid). Additional hydration, dosage reduction and less frequent administration are useful suggestions. [7]

Despite great benefits of antiresorptives in $\mathrm{MM}$, in particular intravenous administration of BPs can lead to medication-related osteonecrosis of the jaws (MRONJ). [6] The diagnostic criteria for MRONJ include a history of bisphosphonate or antiresorptive usage, no history of radiotherapy, and the presentation of exposed or necrotic alveolar bone of maxilla or mandible for more than eight weeks with or without pain. There can also be showed that evidence of regional soft-tissue inflammation or infection, exposed bone with pain, swelling or cutaneous fistula. [8]

MRONJ case results from a combination of multiple systemic and local factors. [9] Events deemed relevant to development of MRONJ involve reduced bone repair, impaired bone turnover, suppressed osteoclast activity, infection, inflammation and impaired angiogenesis. [10] Besides the characteristics of BP and other antiresorptive therapies, high drug potency, the IV administration, and prolonged treatment are the risk factors of MRONJ. [11]

The aim of this study is to report and discuss a MRONJ case within reason incompatible prosthesis in 61-year old female patient diagnosed as multiple myeloma and treated with zoledronic acid by this way to be directive about preventive management.

\section{Case Report}

61-year-old female patient was referred to our department for complaint of difficulty on usage of her prostheses and mild pain at anterior mandibular region. In medical history, it was learned that chemotherapy for MM has been continued for 5 years. The patient was treated with $4 \mathrm{mg}$. zoledronic acid (Zometa, Novartis, NJ, USA) and additionally bortezomib and dexamethasone chemotherapy. The patient and her relatives noted that the patient did not receive radiotherapy. 8 months ago, the left mandibular canine tooth was extracted by a dentist. After soft tissue healed, her old denture was repaired and she continued to use it. Two months later than tooth extraction, the patient started to complain because of mild pain and erythema at anterior mandibular region. An intraoral examination revealed the presence of exposed bone on three areas at anterior mandibular region (Figure 1). The patient was noted that these lesions were available for 4 months. There was no hypoesthesia of the lower lip. About $0.5 \mathrm{~cm}^{2}$ areas of exposed necrotic bone of the mandibular anterior region associated with mucosal inflammation and slight purulent discharge.

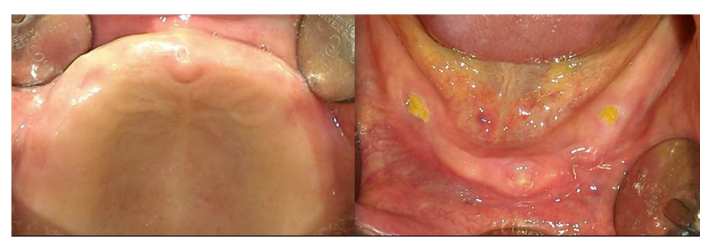

Figure 1. Intraoral Views of Edentulous Patient and Exposed Bone Areas on Mandible.

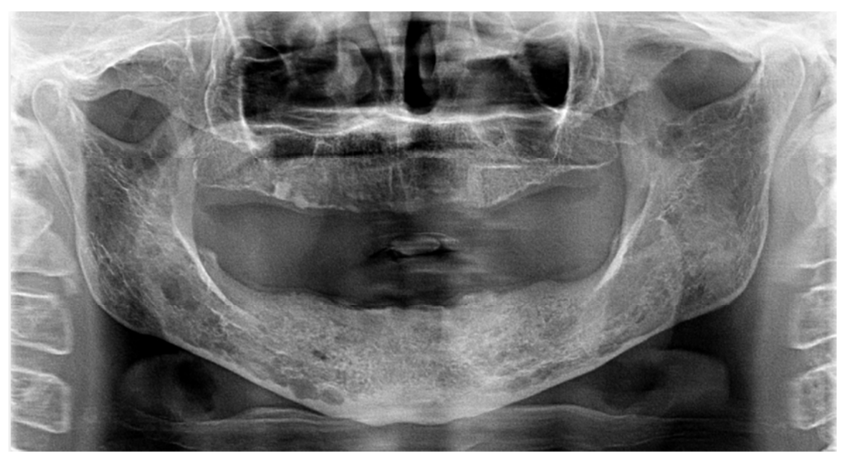

Figure 2. Diffuse Bone Destruction and Increased Bone Trabeculation on Panoramic Graphy.

Routine panoramic radiographs showed that diffuse bone destruction and increased bone trabeculation (Figure 2). Both lateral and postero-anterior cephalometric radiographs showed characteristic punched-out lytic lesions of MM (Figure 3). With these informations, this condition was diagnosed as stage 2 MRONJ induced by incompatible complete removable denture.

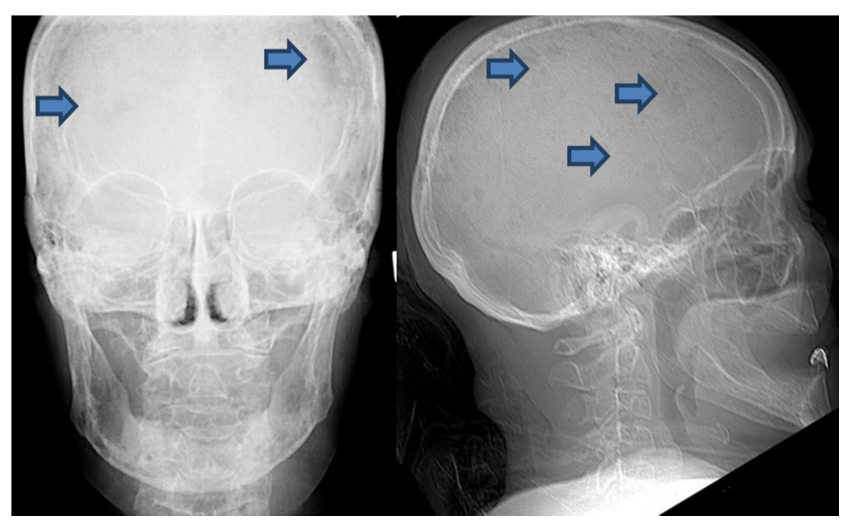

Figure 3. Characteristic Punched-out Lytic Lesions on Skull Graphies.

Firstly, the use of the complete removable denture was only allowed while eating. (Figure 4) For treating pain, swelling and pus, it was decided to oral antibiotic usage. A sample was observed with a swab for cultural analysis. Appropriate antibiotic was prescribed according to antibiogram results. According the results, amoxicillin with clavulanic acid (Augmentin, GlaxoSmithKline) administered until acute inflammation was gone, for this patient, this period was 3 months.

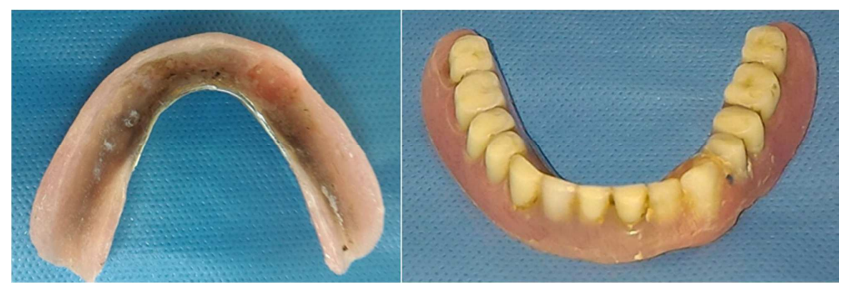

Figure 4. The Incompatible Complete Removable Denture of Patient.

Additionally, debridement and sequestrectomy procedures were made for the MRONJ lesion at the right side. This patient was under follow-up monthly. For the right side, 
complete mucosal healing and for other two region, positive progress was observed at one-month follow-up. (Figure 5). Unhappily, the patient died 2 months after this date.

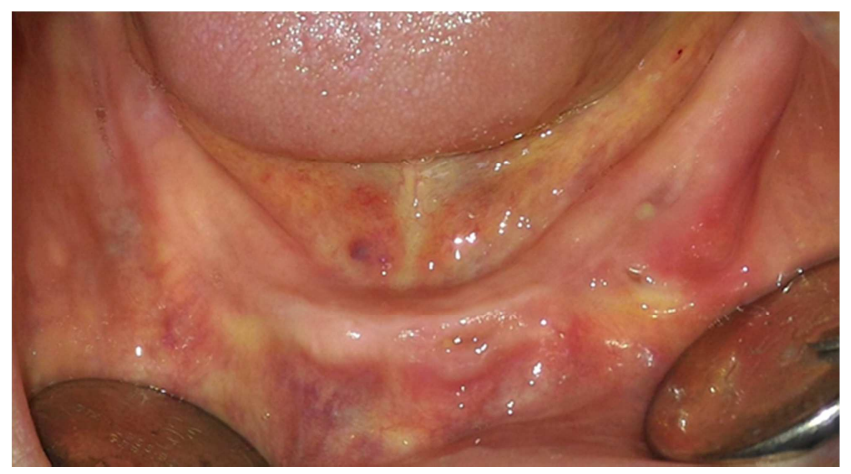

Figure 5. At One-month Follow-up, INTRAORAL Views of the Lesions.

\section{Discussion}

Multiple Myeloma patients are highly appropriate to be treated with bisphosphonates because of their clinical characteristics. The MRONJ has been observed more frequently in MM patients because of the extended duration of exposure. Despite there are some clinical and radiological signs and symptoms can help to clinician, definitive diagnosis of MRONJ is quite difficult. [12] For prevention and management of $\mathrm{MM}$, it is important to make decision that effective strategies will require collaboration between the medical and dental professionals.

Effect of BPs is basically suppressing the proliferation and action of osteoclasts. [13] Pamidronate and zoledronic acid are the most widely used BPs in oncology and hematology, among the different BPs. [14] RANK ligand inhibitors (denosumab) is a novel antiresorptive agent and inhibit osteoclast function. Recently, it is reported that osteonecrosis of jaws cases associated with other antiresorptive (denosumab) and antiangiogenic therapies increase gradually. [15] Therefore, a change was needed to accommodate the term of BRONJ. In 2014, American Association of Oral and Maxillofacial Surgeons (AAOMS) recommended that the term "Bisphosphonate-related Osteonecrosis of The Jaw" (BRONJ) be replaced with the new terminology "Medication-related Osteonecrosis of the Jaw" (MRONJ). Based on the position paper, a definitive diagnosis of MRONJ can be made if all of the criteria are met. These criteria are; 1 . Current or previous medication usage known to cause osteonecrosis of jaws (ONJ) (antiresorptive agents, RANKL inhibitors, VEGFR inhibitors, etc.); 2. Exposed bone in the oral cavity that has persisted for at least 8 weeks; 3 . No previous history of radiation involved to the jaws. [8]

A staging system established which classifies four stages of MRONJ according to the severity of the symptoms and clinical appearance. Stage 0 described as there is no clinical evidence of osteonecrosis due to nonspecific symptoms, radiographic changes and/or clinical findings. Stage 1 described as exposed and necrotic bone in patients with no symptoms or evidence of infection. Stage 2 described as exposed and necrotic bone accompanied by pain, erythema and/or purulent drainage. Stage 3 described as exposed and necrotic bone with one or more of the following; exposed necrotic bone extending beyond the alveolar bone, pathologic fracture, extra-oral fistula, oroantral/oronasal fistula and/or excessive osteolysis reaching to the border of mandible or sinus floor. [8, 16] According to this staging system, a treatment process and recommendations can be planned corresponding to each stage. [17]

Andriani et al. [12] revealed that the most common trigger for MRONJ was dentoalveolar surgery including extractions and following in order of dental implant placement, periodontal disease and dentures. Dental management of patients under current or previous intravenous and oral BF treatment (or with history of treatment) should include close clinically and radiological follow-up at six monthly intervals if there is no clinical lesions or at monthly intervals if there is any necrotic bone area. [18] Additionally clinicians should be careful to eliminate the local risk factors of dental crowns, incompatible dentures or inadequate conservative or restorative treatments. Patients with full or partial dentures should be examined for areas of mucosal trauma. [19] Gridelli [3] suggested that to use of dentures may increase the risk for MRONJ. Elderly patients are more risky about dental problems than their younger counterparts, therefore this is particularly relevant to elderly patients, most of them are over 40 years old. [20]

In conclusion, beside benefits of bisphosphonates and other antiresorptive medications, it should not be ignored that these medications could cause to MRONJ which lead to severe uncomfort and pain for patients. It is important to make decision that effective strategies for diagnosis, prevention and treatment of MRONJ will require a multidisciplinary approach and intervention. It is recommended that patients should be directed to the dental examination for elimination of all dental problems and preventive procedures before usage of these medications.

\section{References}

[1] Parker, S. L., Davis, K. J., Wingo, P. A., Ries, L. A. G., \& Heath, C. W. (1998). Cancer Statistics by Race and Ethnicity. A Cancer Journal for Clinicians, 48(1), 31-48. doi:10.3322/canjclin.48.1.31.

[2] Bird, J. M., Owen, R. G., D’Sa, S., Snowden, J. A., Pratt, G., Ashcroft, J., Behrens, J. (2011). Guidelines for the diagnosis and management of multiple myeloma 2011. British Journal of Haematology, 154(1), 32-75. doi:10.1111/j.13652141.2011.08573.x.

[3] Gridelli, C. (2007). The use of bisphosphonates in elderly cancer patients. The Oncologist, 12, 62-71. doi:10.1634/theoncologist.12-1-62.

[4] Terpos, E., \& Dimopoulos, M. (2005). Myeloma bone disease: pathophysiology and management, Annals of Oncology (May), 1223-1231. doi:10.1093/annonc/mdi235. 
[5] Croucher, P. I. (1998). Bone disease in multiple myeloma. British Journal of Haematology, 103, 902-910. doi:10.1046/j.1365-2141.1998.01082.x

[6] Rugani, P., Acham, S., \& Truschnegg, A. (2010). Bisphosphonate-associated osteonecrosis of the jaws : surgical treatment with ErCrYSGG-laser: case report. Oral Surgery, Oral medicine, Oral Pathology, Oral Radiology, and Endodontology, $110 \quad$ (6), e1-e6. doi:10.1016/j.tripleo.2010.08.013.

[7] Durie, B. G. M., Kyle, R., Belch, A., Bensinger, W., Blade, J., Child, J. A., Webb, I. (n.d.). Myeloma management guidelines a consensus report from the scientific advisors of the international myeloma foundation. The Hematology Journal 4(6), 379-398. doi:10.1038/sj.thj.6200312.

[8] Ruggiero, S. L., Dodson, T. B., Fantasia, J., Goodday, R., Aghaloo, T., Mehrotra, B., \& O'Ryan, F. (2014). American association of oral and maxillofacial surgeons position paper on medication-related osteonecrosis of the jaw-2014 update. Journal of Oral and Maxillofacial Surgery, 72(10), 1938-1956. doi:10.1016/j.joms.2014.04.031.

[9] Peer, A., \& Khamaisi, M. (2014). Diabetes as a risk factor for medication-related osteonecrosis of the jaw. Journal of Dental Research, 94(2), 252-260. doi:10.1177/0022034514560768.

[10] Allen, M. R., \& Burr, D. B. (2009). The Pathogenesis of Bisphosphonate- Related Osteonecrosis of the Jaw : So Many Hypotheses, So Few Data. Journal of Oral and Maxillofacial Surgery. 67(5), 61-70. doi:10.1016/j.joms.2009.01.007.

[11] Saad, F., Brown, J., Van Poznak, C., Ibrahim, T., Stemmer, S., Stopeck, A., Dansey, R. (2012). of the jaw: integrated analysis from three blinded active-controlled phase III trials in cancer patients with bone metastases. Annals of Oncology, 23, 1341-1347. doi:10.1093/annonc/mdr435.

[12] Andriani, A., Petrucci, M., Caravita, T., Montanaro, M., Villiva', N., Levi, A., Gaglioti, D. (2012). Evolution of bisphosphonate-related osteonecrosis of the jaw in patients with multiple myeloma and Waldenstrom's macroglobulinemia : a retrospective multicentric study. Blood Cancer Journal, 2, 1-4. doi:10.1038/bcj.2012.9.

[13] Yi, J. W., Lee, W., Kim, S., Heo, Y., Chae, D., \& Lee, W.
(2014). Effect of Zoledronate on the Expression of Vascular Endothelial Growth Factor-A by Articular Chondrocytes and Synovial Cells : An in Vitro Study. Journal of Bone Metabolism, 21(4), 249-255. doi:10.11005/jbm.2014.21.4.249.

[14] Bedogni, A., Bettini, G., Totola, A., Saia, G., \& Nocini, P. F. (2010). Oral Bisphosphonate - Associated Osteonecrosis of the Jaw After Implant Surgery: A Case Report. Journal of Oral and Maxillofacial Surgery (02782391), 68(7), 16621666. doi:10.1016/j.joms.2010.02.037.

[15] Guarneri, V., Miles, D., Robert, N., Glaspy, J., Smith, I., Thomssen, C., Conte, P. (2010). Bevacizumab and osteonecrosis of the jaw: incidence and association with bisphosphonate therapy in three large prospective trials in advanced breast cancer, 181-188. doi:10.1007/s10549-0100866-3.

[16] Hamadeh, I. S., Ngwa, B. A., \& Gong, Y. (2015). Drug induced osteonecrosis of the jaw. Cancer Treatment Reviews, 41(5), 455-464. doi:10.1016/j.ctrv.2015.04.007.

[17] Bodem, J. P., Kargus, S., Engel, M., Hoffmann, J., \& Freudlsperger, C. (2015). Value of nonsurgical therapeutic management of stage I bisphosphonate-related osteonecrosis of the jaw. Journal of Cranio-Maxillofacial Surgery, 43(7), 1139-1143. doi:10.1016/j.jcms.2015.05.019.

[18] Diel, J. I., Fogelman, I., Al-Nawas, B., Hoffmeister, B., Migliorati, C., Gligorov, J., Aapro, M. S. (2007).

Pathophysiology, risk factors and management of bisphosphonate-associated osteonecrosis of the jaw : Is there a diverse relationship of amino- and nonaminobisphosphonates?. 64(3), 198-207. doi:10.1016/j.critrevonc.2007.07.005.

[19] Campisi, G., Fede, O. Di, Musciotto, A., Casto, A. Lo, Muzio, L. Lo, Fulfaro, F., Gebbia, N. (2007). Bisphosphonate-related osteonecrosis of the jaw (BRONJ): run dental management designs and issues in diagnosis. Annals of Oncology 18 (Supplement 6), 168-172. doi:10.1093/annonc/mdm250.

[20] Demirtaş, N., Aytuğar, E., Kazancioğlu, H. O., \& Erdoğan, E. B. (2015). Multiple myelomalı hastada dental yaklaşım: olgu sunumu. Atatürk Üniversitesi Diş Hekimliği Fakültesi Dergisi, 10, 46-50. doi:10.17567/dfd.93639. 